\title{
Multiaxial life prediction of hydrodynamic plain bearings
}

\author{
Henrik Wünsch ${ }^{1, *}$, Christopher Sous $^{2}$ and Christoph Broeckmann ${ }^{1}$ \\ ${ }^{1}$ Institute for Materials Applications in Mechanical Engineering, RWTH Aachen University, 52062 Aachen, Germany \\ ${ }^{2}$ Institute for Machine Elements and Systems Engineering, RWTH Aachen University, 52062 Aachen, Germany
}

\begin{abstract}
Journal bearings are installed in turbomachinery and in all kind of components related to drive technology. All of these applications expect a high degree of reliability. The functional interaction of shaft, lubricant and bearing shell result in a multiaxial stress state within the bearing lining. However, the life assessment of the bearing material exposed to these multiaxial loads is mostly neglected in the design process. The presented research work formulates a complete methodology for a long-term fatigue assessment, based upon the Quadratic Failure Hypothesis (QFH) This approach satisfies the invariant requirements of coordinate transformation, takes into account positive and negative stresses and is applicable to non-proportional multiaxial loads in 3-dimensional geometric configurations.
\end{abstract}

\section{Introduction}

Journal plain bearings are widely used machine elements. Machine and plant constructors appreciate among other advantages, e.g. towards roller bearings, their efficiency in high-speed applications, their favourable damping characteristics and reliability. As long as journal bearing operate in a hydrodynamic state they are insusceptible to wear phenomena. A wide used parameter for journal bearing design is the specific load $p_{\text {spec }}$, which is the applied load divided by the projected bearing area. This parameter was refused by other authors in the past upon good reason [1,2]. In a dynamically loaded bearing, not the specific load is a parameter for assessing fatigue but rather the multiaxial and often non-proportional stress quantities, induced by the oil film pressure into the lining material. Other influences as mounting stresses and thermal induced stresses in bimetallic designs enhance the multiaxiality of the stress state. In order to overcome the uncertainty of the specific load parameter the design strategy is often to enlarge the projected bearing area to a maximum in order to lowering the specific load. This best practise approach contradicts the ideas of lightweight design and resource efficiency.

To overcome this dilemma other researchers spend great efforts into calculating the oil film pressure in the gap between shaft and bearing under both static und dynamic loads. The solution of the Reynolds equation for lubricating films in which viscosity varies threedimensionally were focus of numerous researches in the last decades and are published in several review papers [3-5]. The output of these calculations are the hydrodynamic pressure (HD) in the lubricating clearance. Extended analysis consider the elasticity of the bearing lining. This elastohydrodynamic (EHD) analysis is refined when temperature terms are taken into account (T-EHD). At this point one can conclude that the hydrodynamic contact with its pressure distribution, clearance height and temperature distribution in the lubrication gap is mostly understood.

However, from this point on, the design chain stops abruptly when it comes to the assessment of fatigue in the lining material. The computed maximum oil pressure is compared with the static compressive strength of the lining material. The German VDI-guideline, e.g., proposes a "thumb rule" for transient operating bearings whereupon the maximum dynamic pressure shall not exceed one sixth of the lining material's compressive strength [6]. For further analysis, the guideline proposes the von Mises criterion. It is well known that for nonproportional loading this approach is often not successful [7]. Until now, there is no reliable study on fatigue in plain bearings known, that considers multiaxial non-proportional stresses. Therefor the authors present here a comprehensive methodology for the fatigue assessment in plain bearings.

\section{Failure Criterion - Quadratic Failure Hypothesis}

Over the past decades, numerous fatigue failure criteria were proposed. The overarching classification is made according to the damage parameter and can be divided up into stress, strain and energy based criteria. [8] The local stress state in a structure can be proportional and non-proportional, which is decisive for the fatigue design process. Based on the common perception, a non-proportional stress state is present when the principal stress axes are rotating during a load cycle. For this case, three groups of different approaches are established and can be subdivided as follows: empirical approach, critical plane approach and integral approach. 
The presented fatigue failure criterion in this paper dates back to the work of Troost and El-Magd [9-11]. They translated the plastic yield criterion for anisotropic materials $[12,13]$ to a fatigue approach. The approach evaluates the fatigue life in a critical oriented volume element. Because of the quadratic term, they named it "Quadratic Failure Hypotheses" (QFH). The general formula can be written as the quadratic potential:

$$
Q_{\mathrm{ijkl}} \sigma_{i j} \sigma_{k l}=2 F
$$

Where $\sigma_{i j}$ is the stress tensor and $Q_{\mathrm{ijkl}}$ is the anisotropy tensor. They interpreted the anisotropy coefficients as the direction-dependent fatigue strength. The rewritten formulation of Eq. (1) by Troost and El-Magd [10] reads as follows:

$$
F=\sum_{\substack{i, j=1 \\ i \leq j}}^{3} \frac{\left(\sigma_{\mathrm{a}}(\mathrm{t})\right)_{\mathrm{ij}}^{2}}{A_{\mathrm{ij}}^{2}}-\sum_{\substack{i, j=1 \\ i \leq j}}^{3} \frac{\left(\sigma_{\mathrm{a}}(\mathrm{t})\right)_{\mathrm{ii}} \cdot\left(\sigma_{\mathrm{a}}(\mathrm{t})\right)_{\mathrm{jj}}}{A_{\mathrm{ii}} \cdot A_{\mathrm{jj}}}=1
$$

In this orientation-dependent equation $\sigma_{\mathrm{a}}(t)$ are the direction- and time-dependent stress amplitudes of the stress tensor and $\boldsymbol{A}$ is the fatigue strength in the respective direction depending on the mean stress $\boldsymbol{\sigma}_{\mathrm{m}}$ acting in this orientation. Based on the basic formulation the criterion was made more convenient and userfriendly by breaking it down to plain stress conditions and by the consideration that a failure under torsional loading accurses always under an angle of $45^{\circ}$. Furthermore the sustainable torsional fatigue strength $A_{i j}$ for $i \neq j$ was formulated as a function of the uniaxial fatigue strength by introducing the ratio $T=\left(\sigma_{f} / \tau_{f}\right)^{2}$ with the fully reversed uniaxial and torsional fatigue strength, which made it applicable for brittle and ductile materials. For the particular case of synchronous and reversed alternating tension-compression load, the criterion was written in its last form as [14]:

$$
\left(\frac{\sigma_{x a}}{\sigma_{f}}\right)^{2}+\left(\frac{\sigma_{y a}}{\sigma_{f}}\right)^{2}-(T-2) \frac{\sigma_{x a} \sigma_{y a}}{\sigma_{f}{ }^{2}}+T\left(\frac{\tau_{x y a}}{\sigma_{f}}\right)^{2}=1
$$

Eq. 3 is for a ratio of $\tau_{f} / \sigma_{f}=1 / \sqrt{3}$ equivalent to the von Mises equivalent stress criterion for alternating stresses. For out-of-phase loading from Eq. 2 a failure value $F_{\mathrm{V}}$ can be derived which is a linear combination of the maximum peak value $\tilde{F}_{\text {max }}$ of the quadratic potential of all orientations and the maximum integral value $\bar{F}_{\text {max }}$ of all orientations.

$$
F_{\mathrm{V}}=\tilde{F}_{\max }\left(\cos \psi_{\mathrm{c}}\right)^{v}+\bar{F}_{\max }\left(\sin \psi_{c}\right)^{w}
$$

By means of the "characteristic principal direction" $\psi_{\mathrm{c}}$ and the "coupling exponents" $v$ and $w$ peak value and integral value are weighted. Thereby it is taken account of the experimental proven fact that loading histories containing shear stresses can be better assessed with the integral value and loading histories in principal stress direction better with the peak value [15]. The characteristic principal direction is calculated by means of the loading stresses:

$$
\psi_{c}=\tan ^{-1} \frac{2\left[\left|\tau_{x y m}\right|+\left(1+3 \frac{\sigma_{y a}}{\sigma_{x a}}\right) \tau_{x y a}\right]}{\left|\sigma_{x m}-\sigma_{y m}\right|+\sigma_{x a}+\sigma_{y a}}
$$

Default values for the coupling exponents are $v, w=2$, which ensure a quasilinear coupling of peak value and integral value [15].

\subsection{Calculation proposal}

The present study is based on the basic considerations of the QFH. Today's computing power allow a numerical solution of the quadratic potential in Eq. 2 for all directions in three-dimensional space. For that reason, complex loading path can be calculated by means of QFH without reducing it to plane stress conditions or analytical solutions in general. Because of the not invariant formulation of $\psi_{c}$ in Eq. 5, the definition was revised. According to the consideration that the peak value is similar to the maximum principal stress criterion the concept of Dietmann [16] and later other authors $[17,18]$ was adapted after which there is beside a critical plane also a critical point of time during a load cycle that is damage relevant. Therefore, the angle $\psi_{\mathrm{c}}$, which is of somewhat empirical nature is replaced by the angle between the orientation of $\tilde{F}_{\max }$ and the principal direction at the time $\tilde{t}_{\text {max }}$. The fundamental steps of the fatigue calculation according to QFH are shown in Fig. 1.

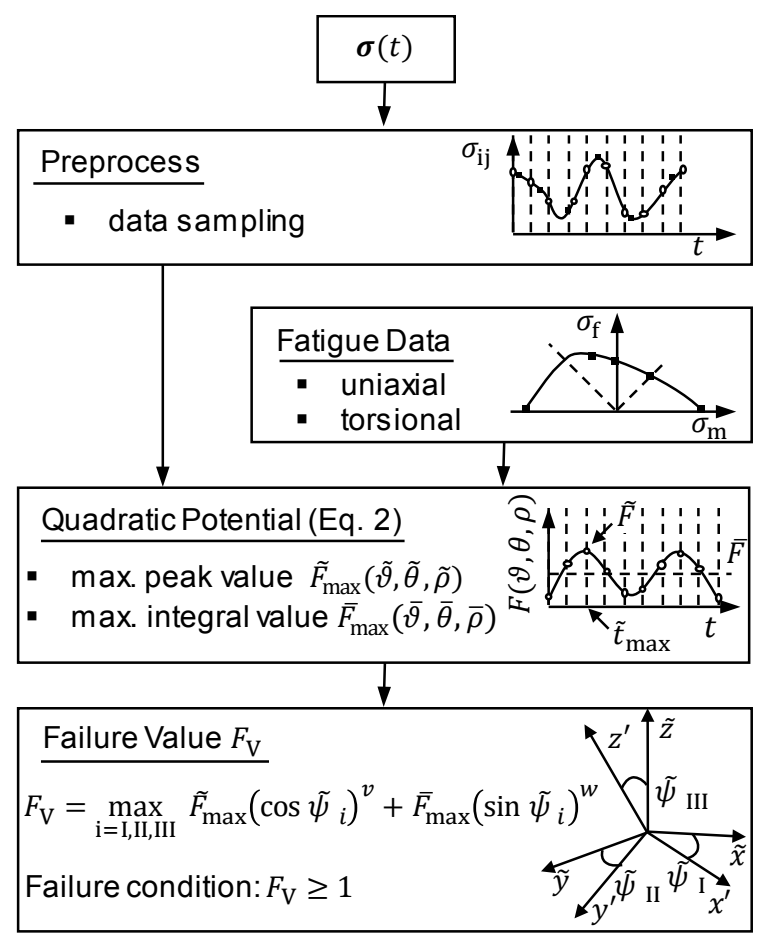

Fig. 1. Flow chart of the calculation method of the QFH.

Input data for the calculation is the time-varying stress tensors for one load cycle. The tensor, which can be the result of a finite element analysis is pre-processed in a initial step. The entries are ordered and sampled in equidistant time intervals. In the next step, the quadratic 
potential is calculated. There for the stress tensor has to be rotated in all spatial directions. The rotation from $\sigma$ to $\boldsymbol{\sigma}^{*}$ through the angles $\vartheta, \phi$ and $\rho$ about the coordinate axis is done using a rotation matrix $\boldsymbol{R}$.

$$
\boldsymbol{\sigma}^{*}(t)=\boldsymbol{R}^{\mathrm{T}} \boldsymbol{\sigma}(t) \boldsymbol{R}
$$

In all spatial directions the entries of the stress tensor can be subdivided in a static part $\boldsymbol{\sigma}_{\mathrm{m}}^{*}$ and a dynamic part $\boldsymbol{\sigma}_{a}^{*}$.

$$
\boldsymbol{\sigma}^{*}(t)=\boldsymbol{\sigma}_{\mathrm{m}}^{*}+\boldsymbol{\sigma}_{\mathrm{a}}^{*}(t)
$$

From the mean stress entries, the sustainable stress amplitudes $\boldsymbol{A}\left(\boldsymbol{\sigma}_{\mathrm{m}}^{*}\right)$ are derived from experimentally determined fatigue strength diagrams for uniaxial and torsional loading. The calculation of the quadratic potential $F$ is performed according to Eq. 2 initially in a rough screening with angle increments of $20^{\circ}$. Via bisection both maxima $\tilde{F}_{\max }$ and $\bar{F}_{\max }$ can be determined. Both maxima are not necessarily located in the same orientation. In order to weight both maxima, the alignment of the axis system of the $\tilde{F}_{\text {max }}$ orientation towards the principal stress directions is decisive. As far as both orientations are identic at the time point $\tilde{t}_{\max }$ when $\tilde{F}_{\text {max }}$ occurs, only the maximum peak value is considered as damage relevant. The greater the misalignment of both orientations the more influence gains the integral value $\bar{F}_{\text {max }}$. Fig. 2 shows the maximum peak value $\widetilde{F}$ as a spatial representation.

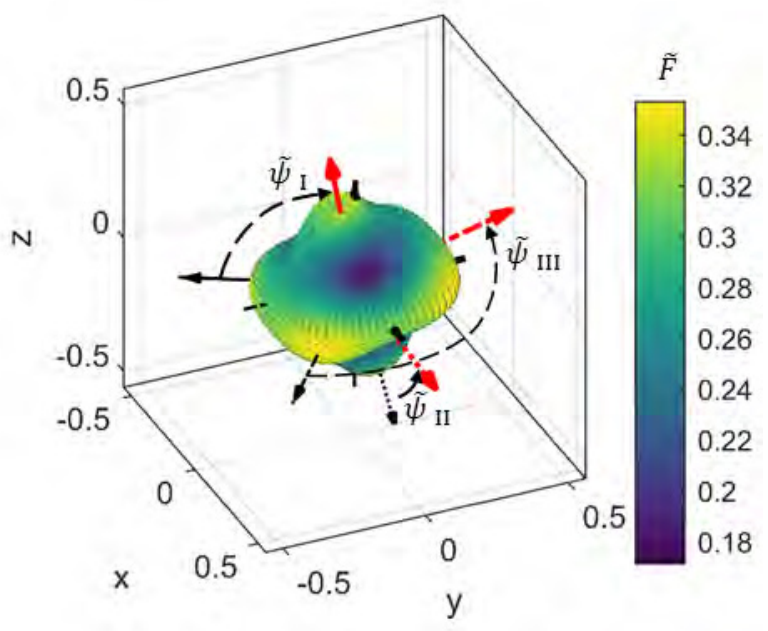

Fig. 2. Maximum peak value $\tilde{F}$ for all spatial directions.

The angles $\tilde{\psi}_{I, I I, I I I}$ represent the misalignment between the orientation of $\tilde{F}_{\max }$ and the principal stress orientation with the case differentiation:

$$
\tilde{\psi}_{i}=\left\{\begin{array}{cc}
\tilde{\psi}_{i} & 0^{\circ} \leq \tilde{\psi}_{i} \leq 90^{\circ} \\
180-\tilde{\psi}_{i} & 90^{\circ}<\tilde{\psi}_{i} \leq 180^{\circ}
\end{array}\right.
$$

The weighting is carried out according to the linear combination:

$$
F_{\mathrm{V}}=\max _{\mathrm{i}=\mathrm{I}, \mathrm{II}, \mathrm{III}} \tilde{F}_{\max }\left(\cos \tilde{\psi}_{i}\right)^{v}+\bar{F}_{\max }\left(\sin \tilde{\psi}_{i}\right)^{w}
$$

The angle $\tilde{\psi}_{i}$ for which the linear combination reaches its maximum is used to calculate the failure value $F_{\mathrm{V}}$. The failure condition is $F_{\mathrm{V}} \geq 1$.

\section{Object of study and material}

The plain bearing under investigation is a fully enclosed journal bearing. This bearing was designed for comparative studies regarding fatigue life on a bearing test-rig. The tested bearings had a width to diameter ratio of 0.4 and an inner diameter of $70 \mathrm{~mm}$. The bearing lining of variable thickness was produced by centrifugal casting. The bearings were subjected to a high sinusoidal load $F_{\mathrm{R}}$, resulting in a pulsating pure compressive load. A sketch of the test-rig and the concept of load application is shown in Fig. 3.

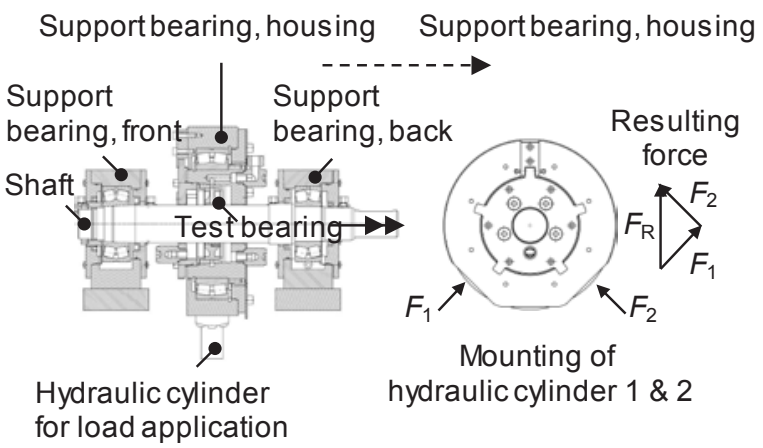

Fig. 3. Journal plain bearing test-rig.

The lining material is a tin-based white metal (SnSb12Cu6ZnAg). Fig. 4 shows a microsection of $\mathrm{SnSb} 12 \mathrm{Cu} 6 \mathrm{ZnAg}$ etched with Iron trichloride. Characteristic is its heterogeneous microstructure with its precipitates $\mathrm{SnSb}$ and $\mathrm{Cu}_{6} \mathrm{Sn}_{5}$, which are embedded in a matrix of saturated tin solid solution. The intermetallic phases, $\mathrm{Cu}_{6} \mathrm{Sn}_{5}$ and $\mathrm{SnSb}$, lead to an increase in static material strength because of precipitation hardening. Antimony causes solid solution hardening, which ensures a reasonable fatigue strength. Traces of silver have a grain refining effect.

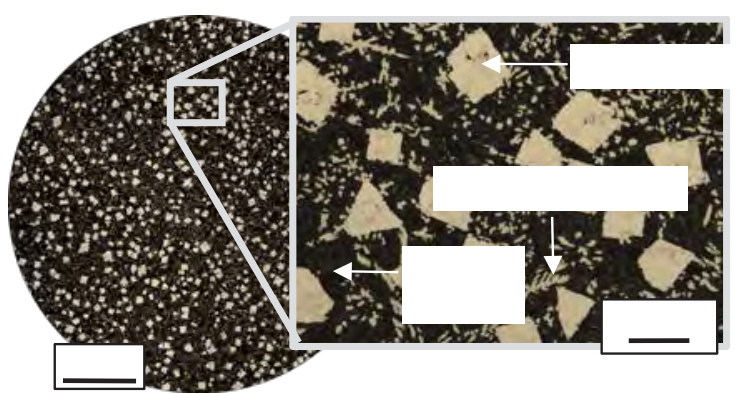

Fig. 4. Microsection of the lining material SnSb12Cu6ZnAg.

Fig. 5 shows the fatigue strength diagram according to Haigh of $\mathrm{SnSb} 12 \mathrm{cu} 6 \mathrm{ZnAg}$ for uniaxial loading with a probability of failure of $50 \%$. The maximum numbers of load cycles was chosen to $10^{7}$ cycles. The load was 
applied in a sinusoidal oscillation at an ambient temperature of $T=100{ }^{\circ} \mathrm{C}$.

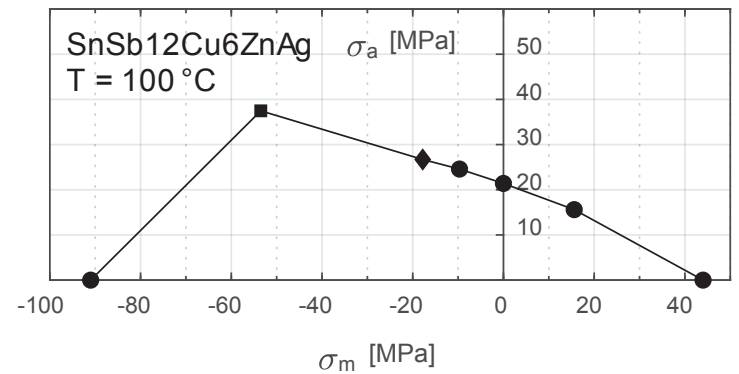

Fig. 5. Fatigue strength diagram according to Haigh of SnSb12Cu6ZnAg for uniaxial loading. - interpolated, - Nesic [19].

Accordingly, Fig. 6 shows under otherwise equal conditions the fatigue strength diagram for torsional loading. In both cases, the solid lines represent the allowable stress amplitude depending on the static mean stress. These are input data for the fatigue calculation below.

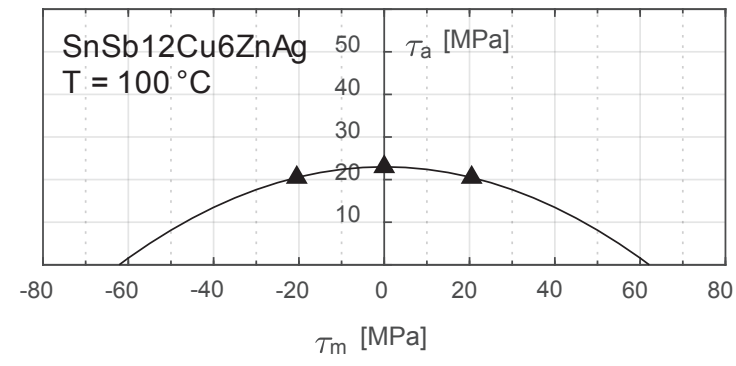

Fig. 6. Fatigue strength diagram according to Haigh of $\mathrm{SnSb} 12 \mathrm{Cu} 6 \mathrm{ZnAg}$ for torsional loading.

\section{Stress analysis}

The geometry and a high number of boundary conditions of this bearing were transferred to a Finite Element Model. The loading of the bearing was calculated by means of the before mentioned T-EHD approach and was imprinted to the FE-Model. A more detailed description of this procedure was published before by Sous et al. in [1]. Fig. 7 shows the bearing under investigation with the definition of the circumferential angle $\theta$, which rotates analogous to the shaft clockwise.

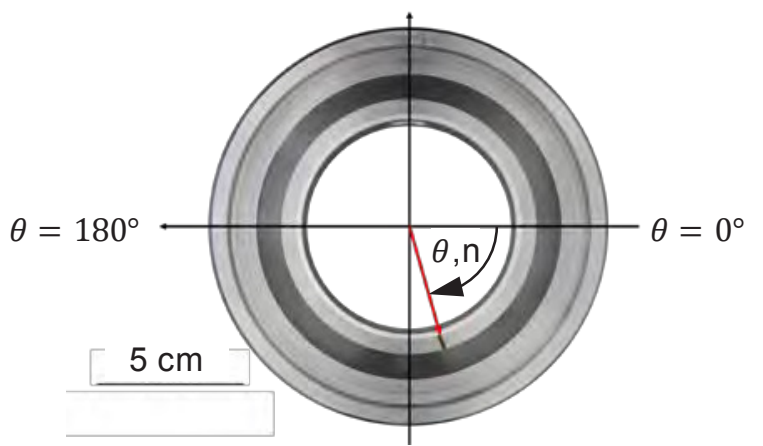

Fig. 7. Test-rig bearing with the definition of the circumferential angle $\theta$.

\subsection{Principal stress in bearing lining}

In order to gain an idea of the stress distribution within the bearing lining Fig. 8 shows the mean principal stress distribution $\sigma_{I, I I, I I I, m} 125 \mu \mathrm{m}$ below the sliding surface of a bearing with a layer thickness of $1 \mathrm{~mm}$ loaded and applied with a specific load of $20 \mathrm{MPa}$. Except in the case of the first mean principal stresses, which act in axial direction, the static mean stresses are compressive. The highest compressive stresses act in radial direction. There are considerable compressive mean stresses in circumferential direction caused by mounting stresses and stresses due to thermal expansion. These are responsible for the asymmetric stress distribution.
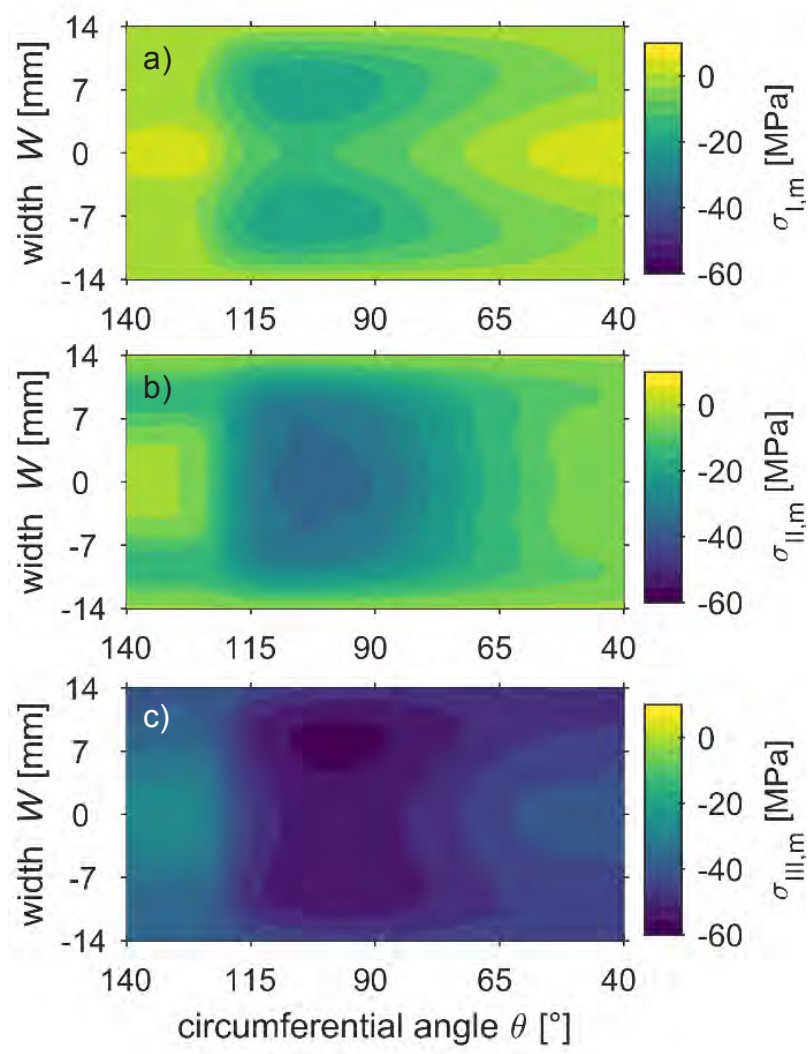

Fig. 8. Distribution of principal mean stresses $\sigma_{I, I I, I I I, m} 125 \mu \mathrm{m}$ below the projected sliding surface for $p_{\text {spec }}=20 \mathrm{MPa}$ and layer thickness of $1 \mathrm{~mm}$.

The amplitudes of the principal stresses $\sigma_{I, I I, I I I, a}$ are shown in Fig. 9. The highest amplitudes act in circumferential direction. This is an indication that a possible crack initiation occurs in direction of the $\sigma_{I I, a}$ direction because of the orientation perpendicular to the free surface. 

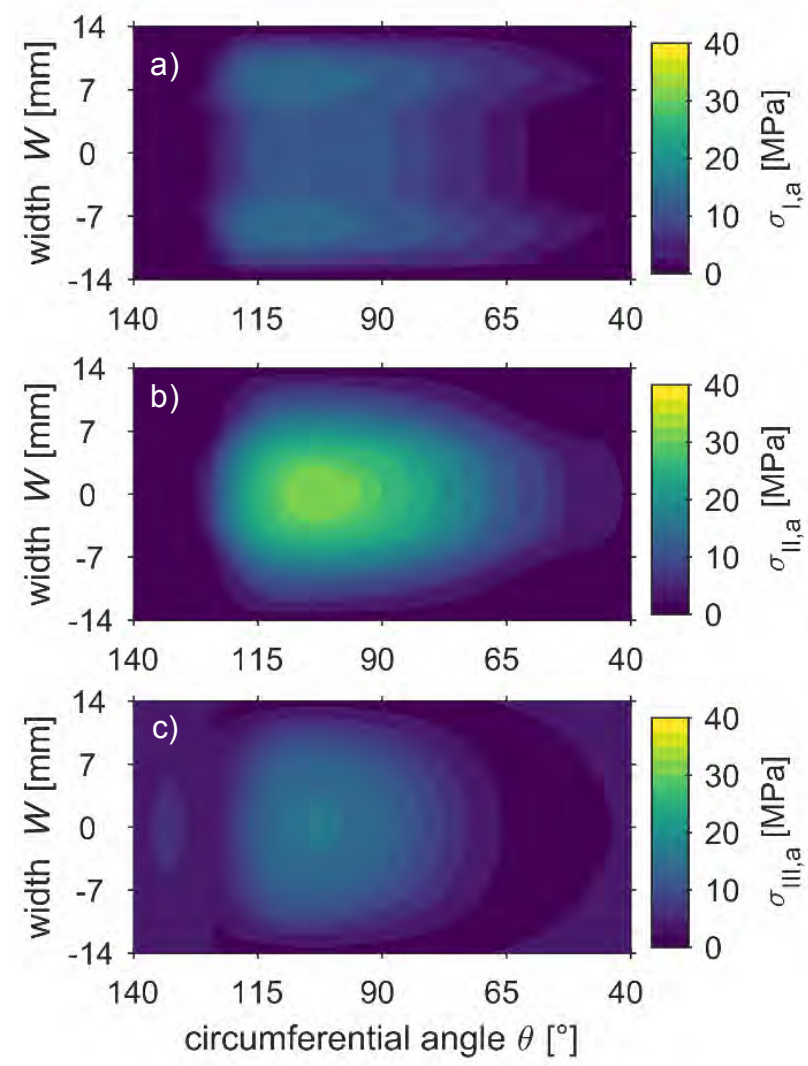

Fig. 9. Distribution of principal stress amplitudes $\sigma_{I, I I, I I I, a}$ $125 \mu \mathrm{m}$ below the projected sliding surface for $p_{\text {spec }}=20 \mathrm{MPa}$ and layer thickness of $1 \mathrm{~mm}$.

Fig. 10 shows the distribution of the maximum shear stresses. The static mean shear stresses increase in rotating direction of the shaft. The time-dependent shear stress amplitudes are of low magnitude and act in areas of low oil-film pressure.
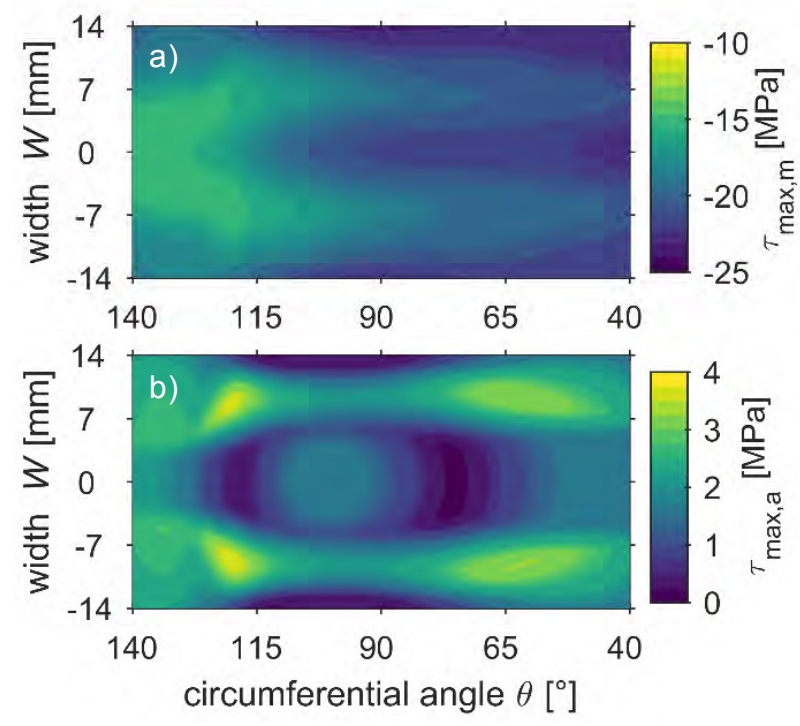

Fig. 10. Distribution of of maximum shear stresses $\tau_{\max , m}$ and $\tau_{\text {max }, a} 125 \mu \mathrm{m}$ below the projected sliding surface for $p_{\text {spec }}=20 \mathrm{MPa}$ and layer thickness of $1 \mathrm{~mm}$.

\subsection{Evaluation of the stress proportionality}

An often-used criterion for proportionality of stress states is the rotation of principal directions. Fig. 11 shows the respective change of the principal direction angles $\Delta \varphi_{I, I I, I I I}$ during one load cycle.
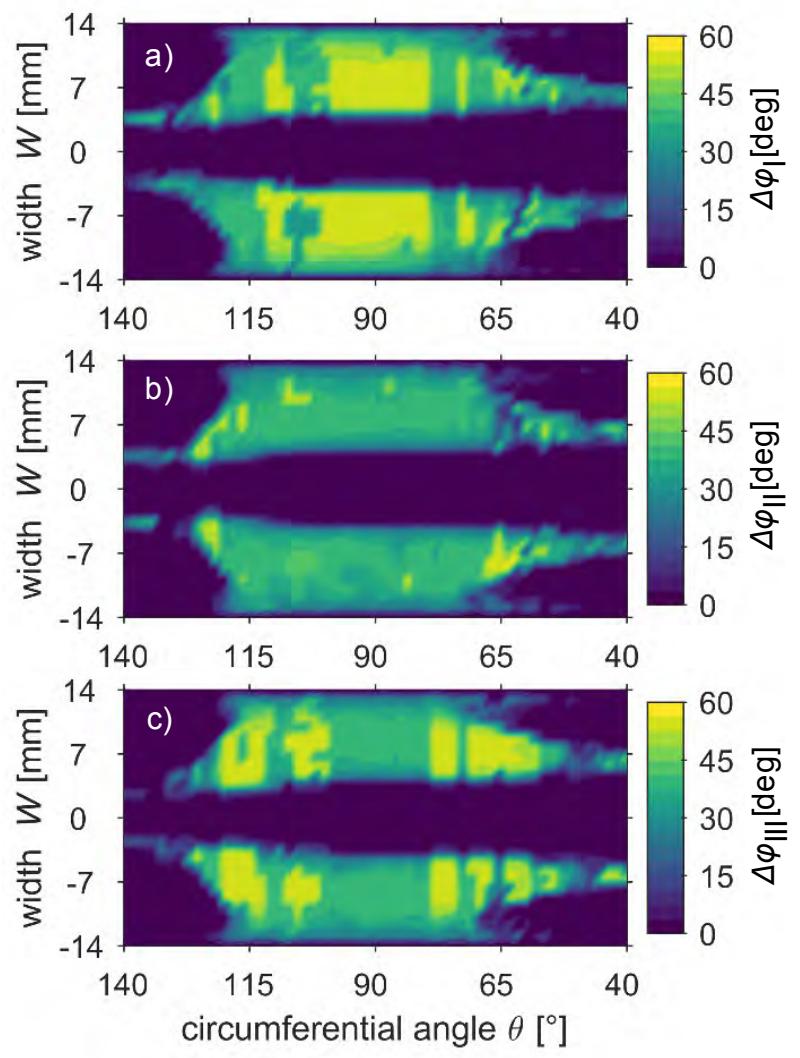

Fig. 11 Change of the principal direction angles $\Delta \varphi_{I, I I, I I I}$ during one load cycle.

According to that, the stress state in the axial middle of the sliding surface where normal stresses are high is proportional. The stresses are non-proportional in areas shifted from the axial middle where normal stresses act in axial direction.

\section{Some calculation examples}

For the same bearing configuration fatigue calculations according to the proposed methodology were carried out. From FE analysis stress tensors for specific loads of 20, 30 and $36 \mathrm{MPa}$ were post-processed via QFH. Fig. 12 shows from a) to c) the distribution of the failure value $F_{\mathrm{V}}$ for increasing specific loads on the projected sliding surface. 

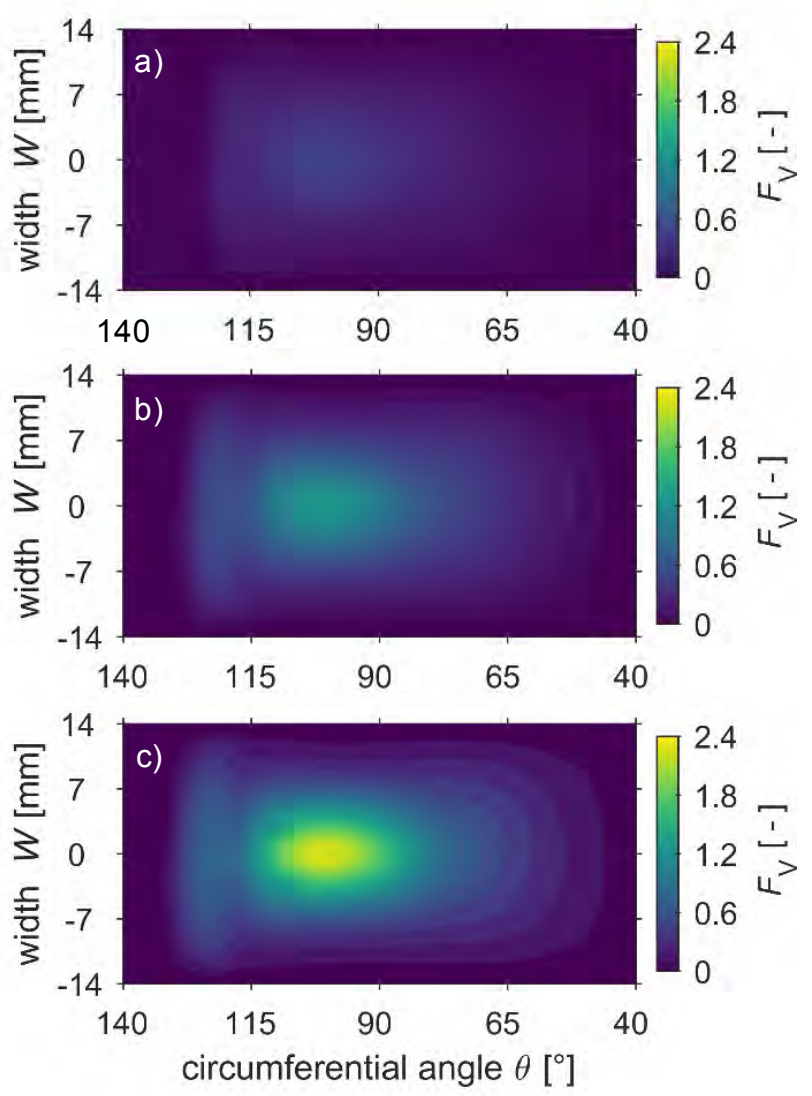

Fig. 12. Distribution and amount of failure value $F_{\mathrm{V}}$ for a) $\left.p_{\text {spec }}=20 \mathrm{MPa}, \mathrm{b}\right) p_{\text {spec }}=30 \mathrm{MPa}$ and c) $p_{\text {spec }}=36 \mathrm{MPa}$ and layer thickness of $1 \mathrm{~mm}$.

For all loading cases the general appearance and distribution of $F_{\mathrm{V}}$ is similar. Logically, the intensity of the failure value increases with increasing specific load. At a circumferential angle of $115^{\circ}$, a discontinuity is apparent. This is the location of the narrowest gap of the oil-film where a transition from compressive stress to tension stress in tangential direction takes place.

In Fig. 13, both summands of the linear combination in Eq. 4 are compared. The exponentials are set to $v=w=1.7$. The peak part clearly predominates the linear combination. The influence of the integral part is marginal.

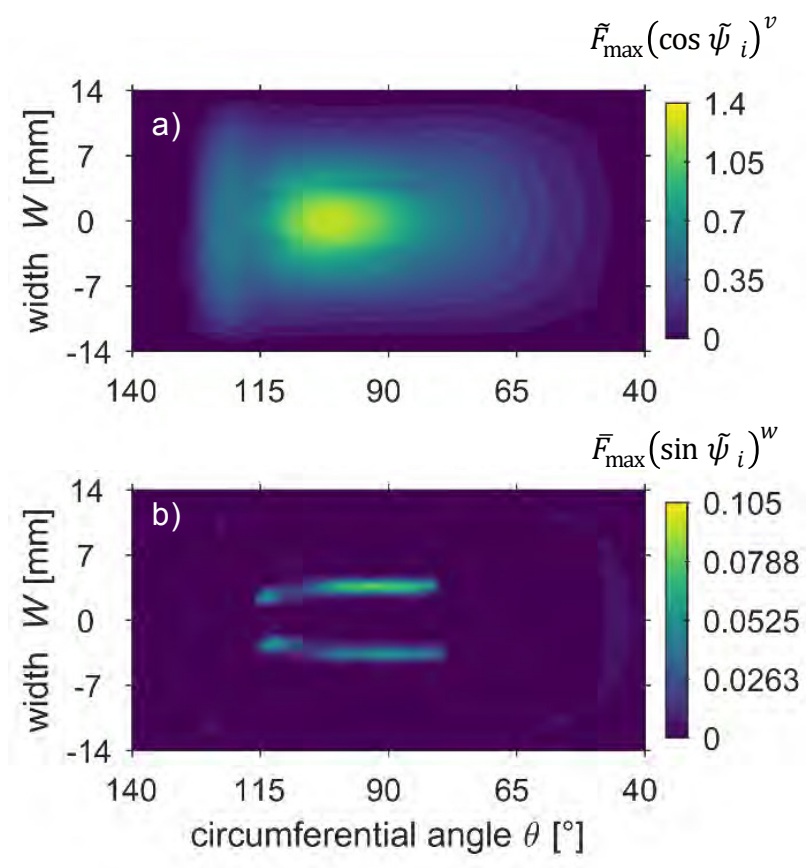

Fig. 13. a) Peak value $\tilde{F}_{\max }$ under consideration of thee weighting factor, b) integral value $\bar{F}_{\text {max }}$ under consideration of thee weighting factor.

The integral part has a minor influence because the angle $\tilde{\psi}_{i}$ according to Eq. 9 is in most areas small. This means automatically that the stress state in these regions is in at least one direction close to the principal stress state.

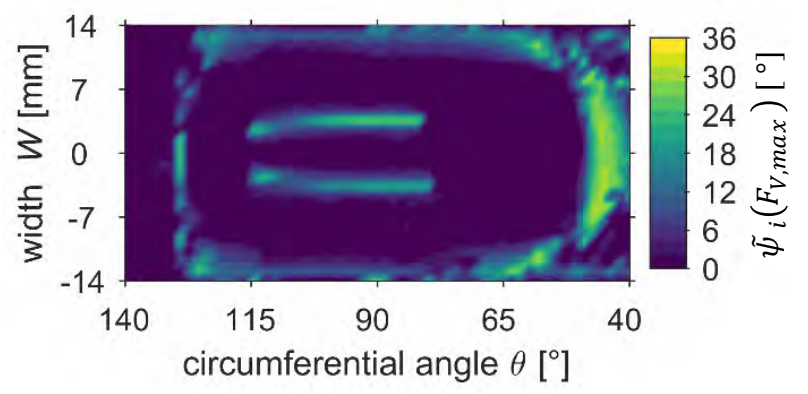

Fig. 13. Weighting angle $\tilde{\psi}_{i}$ for which $F_{\mathrm{V}}$ reaches its maximum.

In accordance to this finding Fig. 14 shows the weighted composition of the failure value $F_{\mathrm{V}}$ at the spot where $F_{\mathrm{V}}$ reaches its absolute maximum at a circumferential angle of $\theta=101.5^{\circ}$.

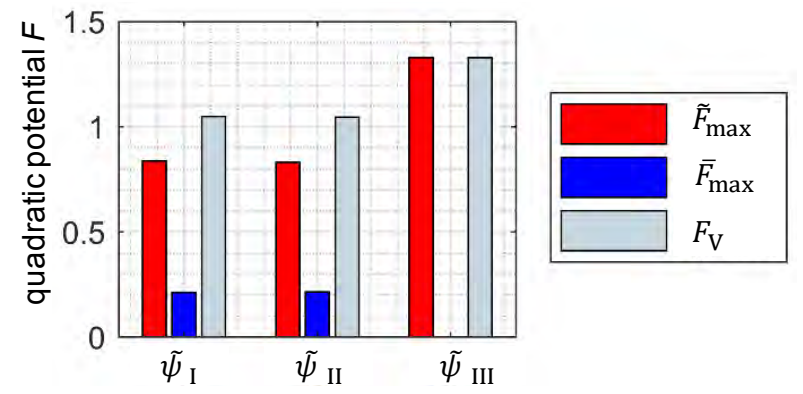


Fig. 14. Peak value $\tilde{F}_{\text {max }}$, integral value $\bar{F}_{\text {max }}$ and failure value $F_{\mathrm{V}}$ depending on the angles $\tilde{\psi}_{I, I I, I I I}$.

Fig. 15 shows the failure value in the axial middle of the sliding surface (at width $W=0$ ). The load cases for a specific load of $36 \mathrm{MPa}$ and $30 \mathrm{MPa}$ exceed the failure condition of $F_{\mathrm{V}} \geq 1$. The area in which the circumferential angle exceeds the failure condition extends with increasing specific load.

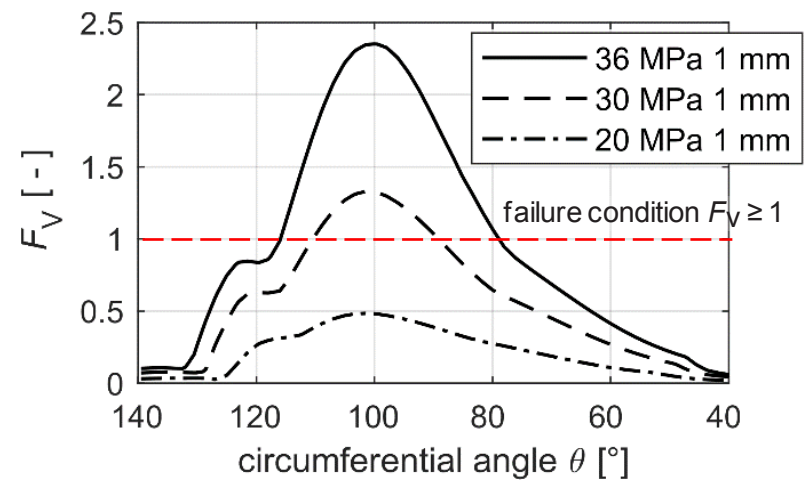

Fig. 15. Failure value $F_{\mathrm{V}}$ for different specific loads.

Therefore, the possibility that a crack initiation takes place at a circumferential angle, which is lower than the one where the maximum is calculated, increases with increasing specific load. Fig. 16 compares the influence of different lining thicknesses on the failure value. It can be shown that a higher layer thickness is more prone to a fatigue damage than a thinner layer. The reason are probably lower compressive mean stresses according to amount and as a consequence thereof, lower sustainable stress amplitudes.

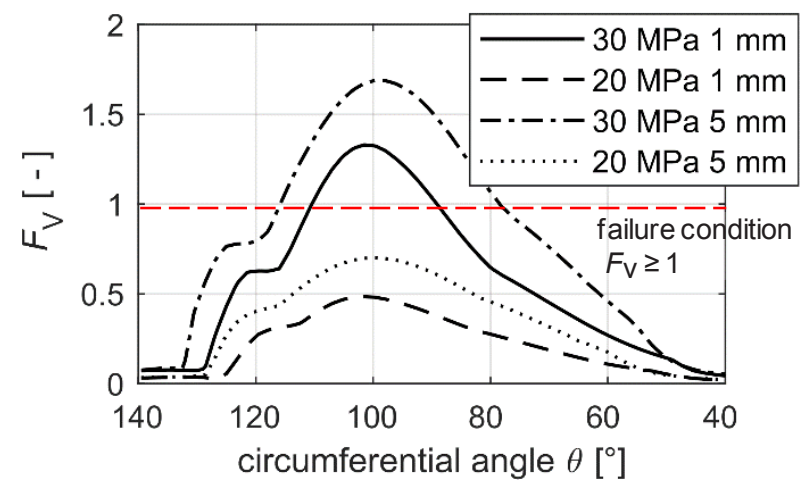

Fig. 16. Failure value $F_{\mathrm{V}}$ for different specific loads and layer thicknesses.

Fig. 17 summaries some results of the described calculation method. The intersection points with the failure condition at $F_{\mathrm{V}}=1$ deliver the maximum allowable specific loads $p_{\text {spec }}$ fort the respective bearing configuration. A fatigue calculation according to Sines [20] is compared to the results of the QFH:

$$
\frac{\sqrt{J_{2, a}}+\left(\sqrt{3} \sigma_{f} / \sigma_{U T S}\right) \sigma_{H, m}}{\tau_{f}}=a
$$

The results of the calculations show a good agreement with the experimentally determined fatigue strength. The Sines criterion, in this case, gives a conservative fatigue life prediction.

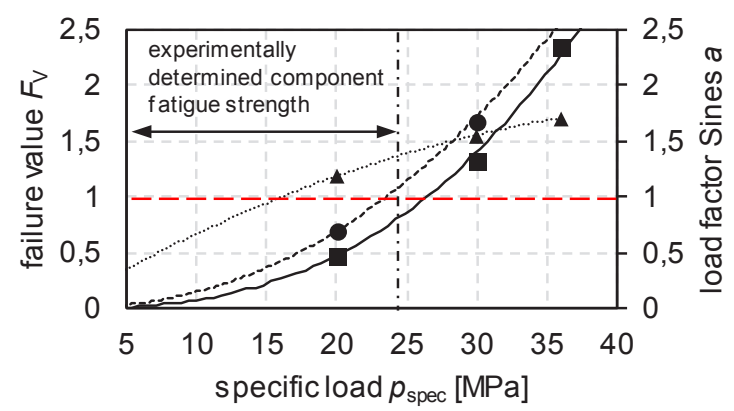

- Layer thickness $1 \mathrm{~mm}$ QFH allowable $\mathrm{p}_{\text {spec: }}$ :

- Layer thickness $5 \mathrm{~mm}, \mathrm{QFH}$

- $26 \mathrm{MPa}, \mathrm{QFH}$

$\Delta$ Layer thickness $1 \mathrm{~mm}$, Sines

- $23 \mathrm{MPa}, \mathrm{QFH}$

- $15 \mathrm{MPa}$, Sines

Fig. 17. QFH calculation for different specific loads and layer thicknesses compared to the Sines criterion and the experimentally determined component fatigue strength

\section{Conclusion and outlook}

The results of the stress analysis and the fatigue assessment based on the QFH provide a unified picture of the fatigue behaviour of journal plain bearings. The regarded boundary conditions including the temperature dependent EHD contact, mounting stresses and thermal expansion within the lining material result in a multiaxial stress state within the sliding layer. However, contrary to the original assumption, the stress state is less nonproportional than expected. This is because shear stresses induced by the viscous oil-film in the lubrication gap are small compared to the induced normal stresses. Superimposed static stresses lead to rotating principal axes in areas of the sliding layer, which are only moderately loaded. According to these observations, the QFH weights the failure relevant mechanisms correctly. The calculated critical orientations considering the anisotropic fatigue strength of the material are consistent with experimental observations, according to which, crack initiation occurs under an angle of $45^{\circ}$ in sliding direction of the shaft. The revised weighting factor between peak and integral part of the failure value is feasible and improves the approach particular in terms of a fully invariant formulation. The calculations have shown that especially normal stresses represented by the peak value $\widetilde{F}_{\text {max }}$ are the fatigue promoting stress quantities.

This is consistent with observations made in SEMinsitu four point bending tests on the lining material, Fig. 18. According to this, the fatigue damage mechanism is as follows: Normal stresses lead to a brittle fracture of $\mathrm{Cu}_{6} \mathrm{Sn}_{5}$ and $\mathrm{SnSb}$. These rod-shaped and cube-shaped phases are cross-linked in the lining volume. Once a fracture in these phases occurs by exceeding a certain threshold, the initiated crack jumps 
to neighbouring precipitations and propagates unstable through the volume. Consequently, in the next stage of the study the presented methodology will be translated from a macroscopic view to a microscopic. With the help of Representative Volume Elements (RVE), stress distributions will be understood on microscopic scale. Because of their general formulation, the QFH seems capable when parameterized with phase specific strength properties to assess fatigue also on microscopic scale.

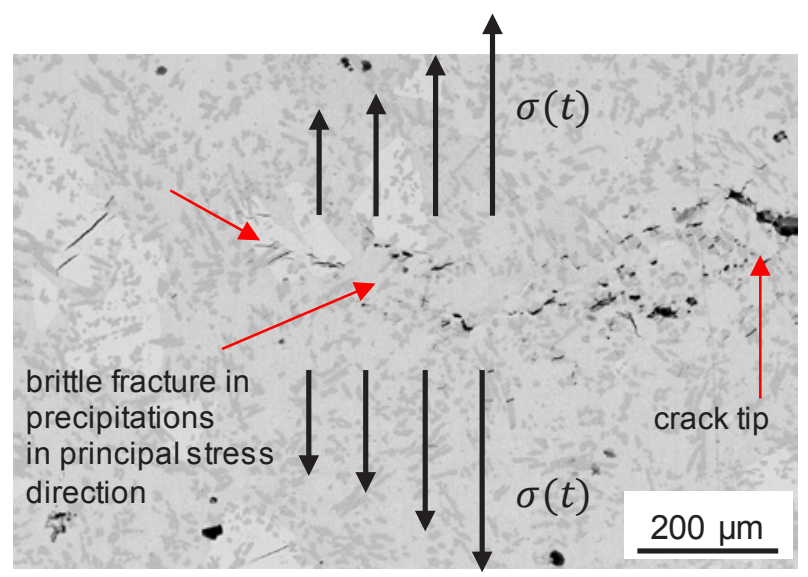

Fig. 18. Mechanism of fatigue crack propagation in front of the crack tip during an insitu four point bending test.

The research for this paper was financially supported by Deutsche Forschungsgemeinschaft (DFG) and Forschungsvereinigung Verbrennungskraftmaschinen e.V. (FVV) in the research project BR 1844/10-2 / JA 1940/4-2 (FVV 1252).

\section{References}

1. C. Sous, H. Wünsch, G. Jacobs, C. Broeckmann, Ind Lubrication and Tribology, 68(3) (2016)

2. F.A. Martin, D.R. Garner, D.R Adams, J. Tribol. 103(1), p. 150 (1981)

3. E. Kutnetsov, S. Glavatskih, Tribology International, 94, pp. 288-305 (2016)

4. M.M. Khonsari, ASLE Transactions, 30(1), pp. 2633 (1987)

5. M. Tanaka, Proceedings of the Institution of Mechanical Engineers, Part J, Journal of Engineering Tribology, 214(1), pp. 107-122 (2005)

6. Verein Deutscher Ingenieure, VDI-Handbuch Produktwentwicklung und Konstruktion, VDI2204 (2007)

7. A. Fatemie, D.F. Socie, Fat Frac Eng Mat Struct, 11(3), pp. 107-122 (1988)

8. T. Łagoda, P. Ogonowski, Mat.-wiss. u. Werkstofftech. 36, No. 9 (2005)

9. A. Troost, O. Akin, F. Klubberg, (1987), Konstruktion, 39 No. 12, pp. 479-488.

10. A. Troost, O. Akin, F. Klubberg, F., Materialwissenschaft und Werkstofftechnik, Vol. 19 No. 12, pp. 406-407 (1988)

11. A. Troost, E. El-Magd, Metall, Vol. 31, pp. 759-764 (1977)
12. R. Hill, The mathematical theory of plasticity, Oxford Univ. Press, (1971)

13. Z. Sobotka, ZAMM 49 (1/2), pp. 25-32 (1969)

14. F. Klubberg, C. Broeckmann, P. Beiss, Materials Testing 54, 9, pp. 569-577 (2012)

15. C. Kaniut, Dissertation, RWTH-Aachen Univerity (1983)

16. H. Dietmann, Z. f. Werkstofffechnik II. of Materials Technology, 6 (1973)

17. K. Dang-Van, Advances in Multiaxial Fatigue, ASTM STP 1191, pp.120-130 (1993)

18. A. Carpinteri, A. Spagnoli, S. Vantadori, International Journal of Fatigue 33, pp. 969-976 (2011)

19. D. Nešić, Dissertation, RWTH Aachen University (2013)

20. G. Sines, Metal Fatigue, pp. 145-169 (1959) 